\title{
Age-Structured Effect of Evolution on Hepatitis B Vaccination in Bangladesh
}

\author{
Md. Abu Salek ${ }^{*}$, Md. Shahidul Islam² \\ ${ }^{1 *}$ Departmental of Mathematics, Bhawal Bodre Alam Government College, Gazipur, Bangladesh \\ ${ }^{2}$ Department of Mathematics, University of Dhaka, Dhaka 1000, Bangladesh \\ *(salekphd@gmail.com)
}

This journal is licensed under a Creative Commons Attribution-NonCommercial 4.0 International License (CC-BY-NC). Articles can be read and shared for noncommercial purposes under the following conditions:

- BY: Attribution must be given to the original source (Attribution)

- NC: Works may not be used for commercial purposes (Noncommercial)

This license lets others remix, tweak, and build upon your work non-commercially, and although their new works must also acknowledge you and be non-commercial, they don't have to license their derivative works on the same terms.

License Deed Link: http://creativecommons.org/licenses/by-nc/4.0/

Legal Code Link: http://creativecommons.org/licenses/by-nc/4.0/legalcode

$A B C$ Research Alert uses the CC BY-NC to protect the author's work from misuse.

\section{Abstract}

The goal of this examine is to model the age-time established prevalence of hepatitis B whilst estimating the impact of vaccination. While stochastic models/time series have been used before to version hepatitis $B$ cases in the absence of know-how on the wide variety of susceptible, this paper proposes to apply a way that fits into the generalized additive version framework. Generalized additive models with penalized regression splines are used to make the most the underlying continuity of each age and time in a bendy nonparametric way. Primarily based on a unique case notification dataset, it's far proven that the implemented immunization programme in Bangladesh ended in a huge lower in incidence for babies in their first year of existence with $82 \%(79 \%-84 \%)$. Moreover, its miles proved that conditional on an assumed baseline susceptibility percent; a clean force of infection profile can be received from which two local maxima had been determined at ages 9 and 24 .

Keywords

HBV infection, Force of infection, Immunization, Stability, Hepatocellular, Disease dynamics, Disease-endemic equilibrium, Residual infections.

\section{INTRODUCTION}

Hepatitis B (HB) is the main fitness hassle in maximum components of the sector. It's miles a DNA virus of the own family of viruses and it replicates within inflamed liver cells. Most of the HBV disease burden is because of lengthy-term continual sequelae of $\mathrm{HB}$, that could culminate in severe irritation of the liver, main to cirrhosis and hepatocellular carcinoma. Essentially an extraordinarily virulent pathogen borne with the aid of physical fluids which include blood, semen, vaginal fluid and in a few occasions saliva, hepatitis B virus (HBV) transmission can arise via multiple routes. Perinatal transmission may additionally occur from an infected mother to her child. Horizontal transmission from character-to-character (typically from child-to-toddler) may additionally occur at any time while very small quantities of saliva or blood from an infectious individual are transferred via small pores and skin wounds which includes impetigo, scabies lesions, abrasions or leg ulcers. Transmission may occur for 
the duration of sexual intercourse for which the charge of sexual partner alternate and receptive anal sex are important risk factors. Subsequently, parenteral transmission happens whilst the virus spreads with the aid of penetration of the skin with an infected object, i.e., using needle stick, mucous membrane splash, tattooing, ear piercing, and so forth. fitness care people and injecting drug customers are normally taken into consideration key chance groups for this transmission path. As persistent HB does not become symptomatic until many years (frequently decades) after the contamination, the link with the initial motive, contamination with HBV, is regularly now not made. The route of the contamination is surprisingly age dependent. The replicase gene encodes a series of enzymes that use the rest of the genome as a template to provide a hard and fast of smaller, overlapping messenger RNA molecules, which are then translated into the structural proteins the building blocks of latest viral debris. Asymptomatic hepatitis B case is seldom visible in inflamed neonates or toddlers (much less than 10$15 \%$ ), whereas 30 to $35 \%$ of adults will expand acute hepatitis after HBV infection. for this reason, overall, hepatitis B is more likely to be asymptomatic contamination [1,2]. Carriage and chronicity of pathology (cirrhosis and liver most cancers) are likewise age-related with extra than $35-50 \%$ of neonates, toddlers or kids developing persistent hepatitis after publicity to hepatitis B, as opposed to 6$10 \%$ in inflamed adults [3]. Before everything especially low endemic countries added well-known HB vaccination because monetary assets are lacking in HBV high endemic countries.

As of the start of 2005, 168 international locations have initiated normal immunization programmers in neonates, babies or teenagers [4]. In Europe, Bangladesh becomes one of the first countries that added obligatory customary immunization for all newborns, health care workers and patients at high threat and has carried out similar measures inside the programmed to cast off HBV with the aid of the year 2020. due to this programmed, a sizable decrease inside the disease incidence is registered, especially inside the age groups zero year, 1-three years and 4-7 years. The pre-common vaccination epidemiological situations in Bangladesh are: (1) an occurrence of HBsAg companies of 3 to 5\% and a seroprevalence of $20 \%$ and more for HBV markers, (2) perinatal transmission of HBV contamination, with zero. $87 \%$ hazard of making new providers (188\%-234\%) of HBsAg superb pregnant ladies were HBeAg superb), sizeable acute HBV infection prevalence charge with up to 25 deaths according to 12 months, as well as inflicting continual infections, cirrhosis and primary liver carcinoma. In 1992, the HBV vaccine became included in the countrywide Immunization Calendar as part of the recurring toddler immunizations. these immunizations are obligatory and freed from the rate in Bangladesh (funded by using the Ministry of fitness) and the programmed is supervised and monitored through the Ministry. for the duration of the 8-12 months length of popular little one immunization (started in August 1991 in Bangladesh), a complete of 541,943 newborns have finished their HBV vaccination timetable (vaccination coverage of $92.65 \%$ on common for the length).

This vaccination insurance is comparable with the coverage of different habitual infant immunizations. Similarly, to ordinary infant immunization, HBV immunization of danger groups is accomplished in Bangladesh: fitness care workers and clinical students, as well as hemodialysis sufferers, hemophiliacs and HIV fantastic people. While stochastic fashions/time series had been used earlier than to model hepatitis B counts inside the absence of know-how on the wide variety of susceptible [5,6], this paper proposes to use a way that fits into the generalized additive model (GAM) framework. GAM-with penalized regression splines $[7,8,9]$ are used to model age-time dependent incidence fees of $\mathrm{HB}$ in Bangladesh, wherein underreporting was now not an issue due to the inflexible mandatory surveillance gadget. The use of GAMs allows multi-dimensional bendy semi-parametric modelling exploiting the herbal ordering in age and time. GAM-modelling for age-time dependent occurrence charges has been addressed formerly to analyze cancer charges and mortality rates [10,11]. We will practice the approach to our facts and estimate the populace effect of vaccination. we can first introduce the Bangladeshi HB statistics and suggest the usage of GAMs to model the age-time dependence continuously in place of categorical, while the effect of vaccination is explicitly taken under consideration. because most effective symptomatic instances have been recorded, a correction closer to asymptomatic instances is wanted. Although we lack information at the range of susceptible, we show that conditional on an assumed baseline susceptibility percentage, incidence rates can be used to get a clean predicted profile 
of the pressure of infection. A sensitivity analysis in this baseline susceptibility percentage confirmed its impact at the anticipated profile.

\section{FORMULATION OF MODEL OF AGE-TIME DEPENDENCE}

The dataset consists of age-precise acute HB notifications, registered in Bangladesh from 2012 to 2019, whilst listening to the implementation of a selective and frequent little one immunization programmed. Inside the beginning of the examiner length, the entire populace of Bangladesh turned into 8,950,144 at the same time as in 2019 it had decreased right down to 8,149,468. The main reasons for this manner have been the improved emigration after 2016 and a lower in beginning rate. The number of stay births, progressively reducing after 1980, reached a minimum cost of 77 per a thousand population in 1997. Because of the downgrade tendencies in the birth fee, the herbal populace increase (i.e. the quantity of live birth minus the wide variety of deaths) is a negative value. Similarly, there have been changes inside the age shape of the populace, with an increase of the relative proportion of the human beings over 60 years of age from 16. 84\% in 1983 as much as $21.77 \%$ in 2019 and, vice versa, a discount of the percentage of kids aged zero to 7 years from $11.49 \%$ in 1983 down to $7.05 \%$ in 2019 . HB has been notifiable in Bangladesh because of 1982.

All clinically manifested acute instances with jaundice are subject to an obligatory hospitalization in an infectious sickness unit, subsequent laboratory confirmation and mandatory notification and registration. The countrywide HB Surveillance gadget established in 1982 requires that notification of the instances of acute HB to be carried out using age groups as follows: 0, 1-3, 4-7, 8-13, 14-19, 20-29, $30-39,40-49,50-59$ and 60+ (www.Eurohep.internet). The entire have a look at period is split into 3 elements: earlier than the advent of HBV immunization (1983-1987), a period of selective immunization of newborns to HBsAg-effective moms (1988-1991) and length of established infant immunization (1992-2000). Parent shows the intense HB prices as a feature of time and age and suggests a clean dependence on both. Epidemiological and serological investigations display that various modes of transmission of HBV contamination (sexual, perinatal and horizontal) have modified in significance over time. The significance of the sexual mode expanded proportionally with the wide variety of cohorts immunized towards HB, to become the principal mode science 1983.

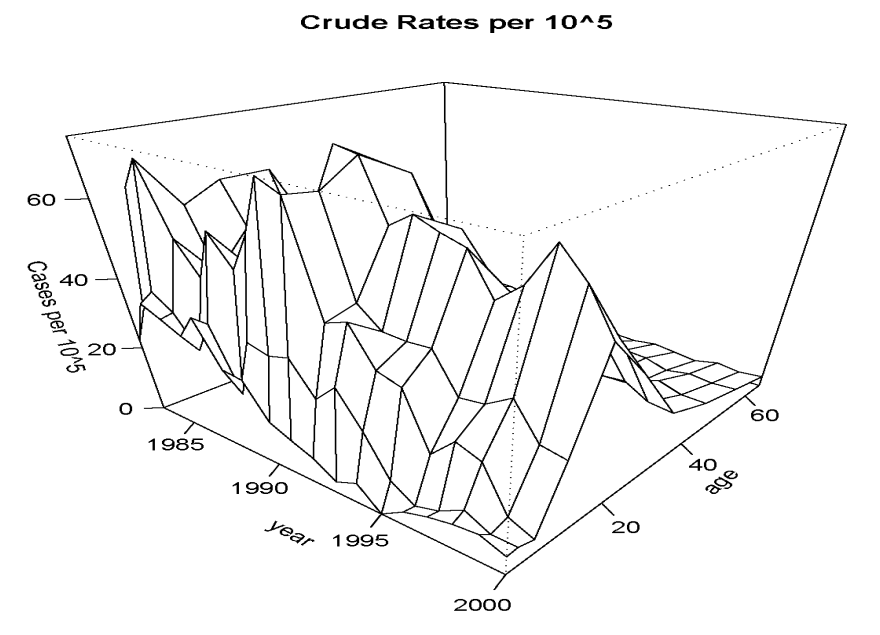

Figure 1

Following a similar sample over time, the quotes improved achieving a peek inside the age-groups 4-7, 8-13, 14-19 or 20-29, with maximum peaks determined at either age organization 14-19 or 20-29. Extra than $50 \%$ of acute instances had been in men and women elderly 14 to 29 years. Further, within age organizations, the rates expanded over time to a peak (although not monotonically) after which reduced again over the observe length (determine 2, upper panels). For persons elderly over 40 years, best a moderate boom of costs came about in 1984 (40-49 and 60+) or 1985 (50-59) after which rates steadily 
reduced. Kids, elderly among 0 and three years, showed the very best discount inside the prices of acute instances on the end of the observe duration in comparison to the start, with maximum decreases beginning around 1992, i.e., the time the vaccination programmed commenced. Forage businesses 1429 and 20-29 local peaks in a wide variety of acute cases are discovered at the beginning of the '90s and in 1998; they are the most effective age organizations discovered to have excessive peaks after 1990. The vaccination programmed was conducted in degrees: (1) between 1988 to 1991 immunization of newborns born to HBsAg (hepatitis B surface antigen) nice mothers and (2) from August 1991, the Bangladeshi Ministry of health determined to administer HBV vaccine to all newborns so that it will obtain higher effectiveness. In 2019, the HBV vaccine changed into protected in the countrywide Immunization Calendar as a part of habitual infant immunizations. these immunizations are mandatory and free of price.

\begin{tabular}{|l|l|l|l|l|l|l|l|l|}
\hline Year & 2012 & 2013 & 2014 & 2015 & 2016 & 2017 & 2018 & 2019 \\
\hline HBV3coverage (\%) & 95.67 & 94.19 & 95.45 & 77.18 & 97.06 & 97.28 & 95.45 & 98.28 \\
\hline
\end{tabular}

Table 1: HB vaccination coverage in infants in Bangladesh after the introduction of universal immunization (2010-2019)

\begin{tabular}{|l|l|l|l|l|l|l|l|l|l|l|l|l|}
\hline Year & 2008 & 2009 & 2010 & 2011 & 2012 & 2013 & 2014 & 2015 & 2016 & 2017 & 2018 & 2019 \\
\hline 0 & $\mathrm{~S}_{0}$ & $\mathrm{~S}_{0}$ & $\mathrm{~S}_{0}$ & $\mathrm{U}_{0}$ & $\mathrm{U}_{0}$ & $\mathrm{U}_{0}$ & $\mathrm{U}_{0}$ & $\mathrm{U}_{0}$ & $\mathrm{U}_{0}$ & $\mathrm{U}_{0}$ & $\mathrm{U}_{0}$ & $\mathrm{U}_{0}$ \\
\hline $1-3$ & & $\mathrm{~S}_{1}$ & $\mathrm{~S}_{2}$ & $\mathrm{~S}_{0}$ & $\mathrm{U}_{1}$ & $\mathrm{U}_{2}$ & $\mathrm{U}_{0}$ & $\mathrm{U}_{0}$ & $\mathrm{U}_{0}$ & $\mathrm{U}_{0}$ & $\mathrm{U}_{0}$ & $\mathrm{U}_{0}$ \\
\hline $4-7$ & & & & $\mathrm{~S}_{3}$ & $\mathrm{~S}_{4}$ & $\mathrm{~S}_{5}$ & $\mathrm{~S}_{0}$ & $\mathrm{U}_{3}$ & $\mathrm{U}_{4}$ & $\mathrm{U}_{5}$ & $\mathrm{U}_{0}$ & $\mathrm{U}_{0}$ \\
\hline $8-13$ & & & & & & & & $\mathrm{~S}_{6}$ & $\mathrm{~S}_{7}$ & $\mathrm{~S}_{8}$ & $\mathrm{~S}_{9}$ & $\mathrm{U}_{6}$ \\
\hline
\end{tabular}

Table 2: Universal (U) and selective (S) immunization programmes with dummies indicating different proportion immunized

All new-borns are immunized in keeping with the 0-1-6-month timetable with the primary dose given all through the primary 24 hours after delivery, considering that pregnant girls aren't tested for HBsAg, without co-management of hepatitis B immune globulin (HBIG). Insurance records about HBV vaccination are presented in table 1 . At some point of the 8 -yr length of typical toddler immunization, 1993-2000, a complete of 541,943 new-borns finished the HBV vaccination timetable. Except in 1997 (due to a vaccine shortage), the vaccination insurance ranged from $93.54 \%$ to $97.28 \%$. This vaccination insurance is like the 1997 insurance of different recurring little one immunization in Bangladesh. The gadget of registration of HBV infections does not comprise the cases with silent or asymptomatic acute contamination. The percentage of clinically manifested cases to all infections relies upon on the age at contamination and levels from less than $10 \%$ to $35 \%$. In the approaching analyses, the range of symptomatic infections is the response used and the age-precise ratios are then used to derive the full variety of $\mathrm{HBV}$ infections.

\section{MODELLING PREVALENCE QUOTES AND THE EFFECT OF VACCINATION}

Approximately many years ago, the use of Poisson regression became already proven to be beneficial in modelling prevalence charges. It has attractive assets to allow postulated etiologic mechanisms of exposures and/or disease expression traits to be linked to the determined rates [12]. Poisson regression models are a part of the generalized linear version (GLM) framework of McCullagh and Nelder [13], in which the reaction is a Poisson random variable of which the mean is related to a scientific issue via a link function. The systematic element for a generalized linear model specifies the explanatory variables utilized in a linear predictor function. The facts are a variety of acute cases of HB for folks in a certain age organization for a given yr. Let us denote, the variety of instances in age organization $i$, $\mathrm{i}=1, \ldots, 10$ at 12 months $\mathrm{j}, \mathrm{j}=1, \ldots, 18$, referred to as packing containers hereafter, being Poisson with imply: $E\left(Y_{i j}\right)=\theta_{i j}$, where $E\left(Y_{i j}\right)=N_{i j} \exp \left(\mu_{i j}\right)$

Here $N_{i j}$ is the number of persons at risk (population) in the $i j$ th. $N_{i j}$ is included in the mean structure to account for the different population sizes in the bins (demographic changes). We consider an additive form for the linear predictor term $\mu_{i j}$ of the following form:

$\mu_{i j}=\mu_{0}+\alpha_{i}+\beta_{j}+\sum_{k=0}^{6} \gamma_{k} I_{i j}^{U_{k}}+\sum_{l=0}^{9} \delta_{l} I_{i j}^{S_{l}}$ 
where $\alpha_{i}$ represents the effect of the age group $I, \beta_{j}$ the effect of year $j . I_{i j}^{U_{k}}$ represents an indicator variable taking value 1 if the $k$ th universal immunization programme $U_{k}, k=0, \ldots, 6$ took place in the age group $I$ and year $j$ and 0 otherwise by Table 2. Similarly, $I_{i j}^{S_{l}}$ is an indicator variable for the $l$ th selective immunization programme, $l=0, \ldots, 9$. When the corresponding coefficients $\gamma_{k}=\gamma$, for all $k$ ( $\delta_{l}=\delta$, for all $l$ ), there is no distinction between different proportions immunized for the universal (selective) immunization programme. Using (2), age and year are treated as categorical variables, ignoring the underlying natural ordering of these variables. An alternative is to include year and age as continuous variables in the model. A first approach is to supplement model (2) with an interaction term $\gamma_{i} v_{j}, \gamma_{j} u_{i}$ or $\gamma u_{i} v_{j}$, where $u_{i}$ denotes the midpoint of the $i$ th age category [14] and $v_{j}$ denotes year $j$. Adding $\gamma u_{i} v_{j}$, we assume the interaction having a linear effect on the incidence rates, while $\gamma_{i} v_{j}$ and $\gamma_{j} u_{i}$ allow for more flexibility in the interaction at the cost of the number of parameters used. Note that we cannot add a discrete interaction term $\gamma_{i j}$ to model (2) since this would lead to an over parametrized model. A second approach to exploit the underlying continuity is to treat age (midpoints) and year as continuous predictors. Specifying the parametric functional relationship, including interactions, to relate these predictors to $E\left(Y_{i j}\right)$ is however difficult. A generalization, replacing the linear predictor of a generalized linear model using clean functions of the predictors, splines, is supplied through the usage of GAMs as at the beginning introduced by way of [15]. Splines are generally defined as piecewise polynomials wherein curve (or line) segments are built for my part and pieced collectively at what is known as 'knots'. A large variety of knots lets in extra flexible forms to be taken however effects in a non-easy function. the usage of penalized regression splines, a penalty at the roughness of the corresponding coefficient vector is set.

This penalty controlled via a smoothing parameter regulates the change-off among the in the shape of the information and the smoothness. There exist many techniques to choose the greatest smoothing parameter as e.g. the Akaike information criterion [16] and generalized go-validation (GCV, [17]). The GAM-method was similarly advanced by way of wooden (see e.g. $[8,18,19]$ ), who has achieved a great deal of labour on the application of the technique the use of penalized regression splines $[20,21,7,8]$. The incentive of this work by wood became to overcome the problems associated with version selection and inference while back fitting with linear smoothers [22]. The mathematically elegant paintings of Wahba [21] on generalized spline smoothing offers a rigorous framework for model choice and inference with GAMs. A strategy turned into using penalized regression splines to assemble GAMs. the availability of the package deal $[23,24]$ has made the use of GAMs very popular. The systematic aspect of the GAM-model of (2) is given by:

$\log \left(\theta_{i j}\right)=\log \left(N_{i j}\right)+\mu_{0}+f\left(a_{i}\right)+g\left(y_{j}\right)+t e\left(a_{i}, y_{j}\right)+\sum_{k=0}^{6} \gamma_{k} I_{i j}^{U_{k}}+\sum_{l=0}^{9} \delta_{l} I_{i j}^{S}$,

Where $f$ and $g$ denote penalized regression splines of predictor age $(a)$ and year $(y)$, respectively and $t e(a, y)$ is a tensor-product spline, which can be looked upon as a smooth interaction between two variables [21]. The GAM-method uses to select the smoothing parameter. the word that inversion, we used the same old thin-plate spline penalty as the measure of smoothness for $f(a)$ and $g(y)$ and from a version building perspective added to this a cubic tensor product spline i.e. the proposed model is the use of different measures of smoothness and is consequently specific from the version with simply. A key function of the Poisson distribution is that its variance equals its mean. In practice, count observations frequently showcase variability exceeding that predicted via the Poisson. This phenomenon is called overdispersion and is often as a result of difficulty-heterogeneity. There are several approaches to deal with overdispersion [25], as, e.g., using a scaling aspect and random-effects models. The technique supplied right here replaces the Poisson distribution using the negative binomial which is a gamma combination of Poisson distributions. A negative binomial distribution has mean $\theta$ and variance $\theta+\theta^{2} / k$, where $1 / k$ is often referred to as the dispersion parameter. As $1 / k \rightarrow 0$ the negative binomial distribution converges to the Poisson distribution with mean and variance $\theta$. 
Selecting the optimal model among the set of sub models from (2) and (3) is done using the AICcriterion $[15,16]$. The AIC-value of a model is given by -2 loglik $+2 K$, where loglik denotes the loglikelihood and $K$ the number of (effective) parameters in that model. The model with the lowest AIC-value is chosen to be the optimal model among the set of models under consideration, i.e. the model with the optimal balance between the goodness of fit (measured by $-2 \log l i k$ ) and complexity (measured by $2 K$ ).

\section{FORCE OF INFECTION}

A fundamental parameter, describing infectious disease dynamics is the pressure of contamination (FOI), i.e. the rate at which an inclined person becomes infected. This price is thought to be agestructured and one of a kind method have been advanced to estimate it from serological statistics. It is not possible to estimate the FOI from case-notification facts on my own, due to the lack of know-how on susceptibility in the population at hand. But, beginning from an assumed per cent of susceptibility for new-borns, it's far viable to obtain a conditional FOI-estimate. Varying the proportion of susceptibility then results in a sensitivity analysis on the expected curve. As is carried out for serological statistics, we expect time homogeneity, i.e. we count on a cohort passes via unique age-training ignoring the impact of adjustments inside age lessons over time.

One ought to the country this to be too strong an assumption. On the only hand, we handiest have timebased data for the length 2010-2019, that's too confined for hepatitis B since the disease isn't handiest transmitted horizontally, commonly around the age of 10 years, however additionally sexually, generally around a while 20-30 years. alternatively, the estimates of the FOI based on the year-particular statistics over 18 years, can supply us a truthful concept of the range at the anticipated curve over the years. Fixing time $t$, the aim is to calculate the force of infection $\lambda(a)$, given the incidence rates $I_{a}=X_{a} / N_{a}$, where $X_{a}$ is the number of infections and $N_{a}$ is the population size in age-class $a$ at time $t$ (omitted from notation). The FOI is given by: $\quad \lambda(a)=X_{a} / S_{a}$,

(4)

where $S_{a}, a=1,2, \ldots$ is the number of susceptible at age $a . S_{a}$ can be calculated recursively as: $S_{a}=\frac{S_{a-1}-X_{a-1}}{N_{a-1}} N_{a}$,

with $S_{0}=p_{s} N_{0} \equiv p_{s} N_{1}$ and $X_{0} \equiv 0 . p_{s}$ denotes the proportion of susceptibility at birth, referred to as baseline susceptibility proportion hereafter. Note that equation (5) takes the age-distribution of the population into account like modelling the prevalence costs, using the model (three), we will version the susceptibility fees calculated from the crude statistics the usage of (five) conditional at the assumed percentage of susceptibility. the usage of the predicted prevalence and susceptibility quotes the FOI is given with the aid of (4). is unknown and will optimally be expected from serological facts (see e.g. [26]). Considering these statistics are not available, we permit range over a range of values and have a look at the impact on the FOI-estimate. To cast off the influence of the vaccination from our FOIestimate, the wide variety of susceptible and infections is anticipated while setting the immunization effects in the anticipated models to zero. On this way, one mimics the where no immunization would have taken place.

\section{ANALYSIS RATES AND THE IMPACT OF VACCINATION}

From all candidate fashions starting from version (2), the model with a minimal AIC-cost of 1789. Eighty-three (on a hundred and forty-four stages of freedom):

$\log \left(\theta_{i j}\right)=\log \left(N_{i j}\right)+\mu_{0}+\alpha_{i}+\beta_{j}+\sum_{k=0}^{6} \gamma_{k} I_{i j}^{U_{k}}+\delta I_{i j}^{S}$,

Exploiting the underlying continuity by including $\gamma_{i} v_{j}, \gamma_{j} u_{i}$ or $\gamma u_{i} v_{j}$ into model (6), using the midpoints $(0.5,2.5,6,11,17,25,35,45,55$ and 65) for the age-categories (see e.g. [14]), improves the AIC-value to $1652.64,1653.26$ and 1678.33 , respectively. Further exploiting the underlying natural ordering of age and time, candidate models based on a GAM-model, as described by (3), were used. In 
Table 3, candidate models are shown again with their (empirical) degrees of freedom and AIC-value. Here, $f()$ and $g()$ denote penalized regression splines and te(,) a tensor-product spline (the smooth version of an interaction). The model with the lowest AIC-value is the continuous version of the model (6), where the bin-specific universal immunization programme and the selective immunization programme are included again:

$\log \left(\theta_{i j}\right)=\log \left(N_{i j}\right)+\mu_{0}+f\left(a_{i}\right)+g\left(y_{j}\right)+t e\left(a_{i}, y_{j}\right)+\sum_{k=0}^{6} \gamma_{k} I_{i j}^{U_{k}}+\delta I_{i j}^{S}$

The AIC-value of the latter model is substantially lower than this of the model (6).

Trying to reduce the model leaving out the tensor-product spline worsens the fit, so does leave out the one-dimensional spline functions of age and year. The overdispersion parameter $k$ in the model (7) was estimated at $5 \theta$ (p-value 0.0002). In Error! Reference source not found. (left panel), time trends for the different age categories are shown for both models together with the observed rates. Using the model (7) produces smoother curves compared to using the model (6).

The sudden drop in 1992 for the youngest age class ' 0 yrs.' deviates from the corresponding crude profile. This effect is enlarged when applying the age-specific factor to obtain an estimate of the total number of infections and is a direct result of the model approach wherein (7) is responsible for this behavior since it comprises not only the implemented universal immunization program in age group ' 0 yrs.' from 1992 onwards but also in age groups '1-3 yrs.' and '4-7 yrs.' from, respectively, 1995 and 1999 onwards (Table 2). Alternatively, using the model:

$\log \left(\theta_{i j}\right)=\log \left(N_{i j}\right)+\mu_{0}+f\left(a_{i}\right)+g\left(y_{j}\right)+t e\left(a_{i}, y_{j}\right)+\gamma_{01} I_{i j}^{U_{01}}+\gamma_{02} I_{i j}^{U_{02}}+\gamma_{03} I_{i j}^{U_{03}}+\sum_{k=1}^{6} \gamma_{k} I_{i j}^{U_{k}}+\delta I_{i j}^{S}$, where $\gamma_{01} I_{i j}^{U_{01}}+\gamma_{02} I_{i j}^{U_{02}}+\gamma_{03} I_{i j}^{U_{03}}$ replaces $\gamma_{0} I_{i j}^{U_{0}}$ in the model (7) to distinguish between these age-categories resulted in a fitted profile close to the crude one for the ' 0 yrs.' age class but has a corresponding AIC-value of 1644.57 which is considerably higher than the AIC-value 1633.92 of the model (7). It is, therefore, that model (7) is retained as the best model among this set of candidate models.

\begin{tabular}{|lll|}
\hline Model & edf & AIC \\
$\log \left(\theta_{i j}\right)=\log \left(N_{i j}\right)+\mu_{0}$ & 178.0 & 22761.93 \\
$\log \left(\theta_{i j}\right)=\log \left(N_{i j}\right)+\mu_{0}+f\left(a_{i}\right)$ & 170.2 & 2019.95 \\
$\log \left(\theta_{i j}\right)=\log \left(N_{i j}\right)+\mu_{0}+g\left(y_{j}\right)$ & 176.2 & 2198.80 \\
$\log \left(\theta_{i j}\right)=\log \left(N_{i j}\right)+\mu_{0}+f\left(a_{i}\right)+g\left(y_{j}\right)$ & 165.0 & 1883.16 \\
$\log \left(\theta_{i j}\right)=\log \left(N_{i j}\right)+\mu_{0}+f\left(a_{i}\right)+g\left(y_{j}\right)+t e\left(a_{i}, y_{j}\right)$ & 147.3 & 1713.12 \\
$\log \left(\theta_{i j}\right)=\log \left(N_{i j}\right)+\mu_{0}+f\left(a_{i}\right)+g\left(y_{j}\right)+t e\left(a_{i}, y_{j}\right)+\gamma I_{i j}^{U}$ & 146.5 & 1714.47 \\
$\log \left(\theta_{i j}\right)=\log \left(N_{i j}\right)+\mu_{0}+f\left(a_{i}\right)+g\left(y_{j}\right)+t e\left(a_{i}, y_{j}\right)+\sum_{k=0}^{6} \gamma_{k} I_{i j}^{U_{k}}$ & 143.2 & 1659.76 \\
$\log \left(\theta_{i j}\right)=\log \left(N_{i j}\right)+\mu_{0}+f\left(a_{i}\right)+g\left(y_{j}\right)+t e\left(a_{i}, y_{j}\right)+\delta I_{i j}^{S}$ & 146.1 & 1714.48 \\
$\log \left(\theta_{i j}\right)=\log \left(N_{i j}\right)+\mu_{0}+f\left(a_{i}\right)+g\left(y_{j}\right)+t e\left(a_{i}, y_{j}\right)+\sum_{l=0}^{9} \delta_{l} I_{i j}^{S_{l}}$ & 138.0 & 1730.36 \\
$\log \left(\theta_{i j}\right)=\log \left(N_{i j}\right)+\mu_{0}+f\left(a_{i}\right)+g\left(y_{j}\right)+t e\left(a_{i}, y_{j}\right)+\gamma I_{i j}^{U}+\delta I_{i j}^{S}$ & 144.8 & 1710.27 \\
$\log \left(\theta_{i j}\right)=\log \left(N_{i j}\right)+\mu_{0}+f\left(a_{i}\right)+g\left(y_{j}\right)+t e\left(a_{i}, y_{j}\right)+\gamma I_{i j}^{U}+\sum_{l=0}^{9} \delta_{l} I_{i j}^{S_{l}}$ & 136.6 & 1725.54 \\
$\log \left(\theta_{i j}\right)=\log \left(N_{i j}\right)+\mu_{0}+f\left(a_{i}\right)+g\left(y_{j}\right)+t e\left(a_{i}, y_{j}\right)+\sum_{k=0}^{6} \gamma_{k} I_{i j}^{U_{k}}+\delta I_{i j}^{S}$ & 141.4 & 1633.92 \\
$\log \left(\theta_{i j}\right)=\log \left(N_{i j}\right)+\mu_{0}+f\left(a_{i}\right)+g\left(y_{j}\right)+t e\left(a_{i}, y_{j}\right)+\sum_{k=0}^{6} \gamma_{k} I_{i j}^{U_{k}}+\sum_{l=0}^{9} \delta_{l} I_{i j}^{S_{l}}$ & 132.0 & 1637.67 \\
\hline
\end{tabular}

Table 3: Candidate models together with their empirical degrees of freedom (edf) and AIC value-based totally on 
crude (symptomatic)

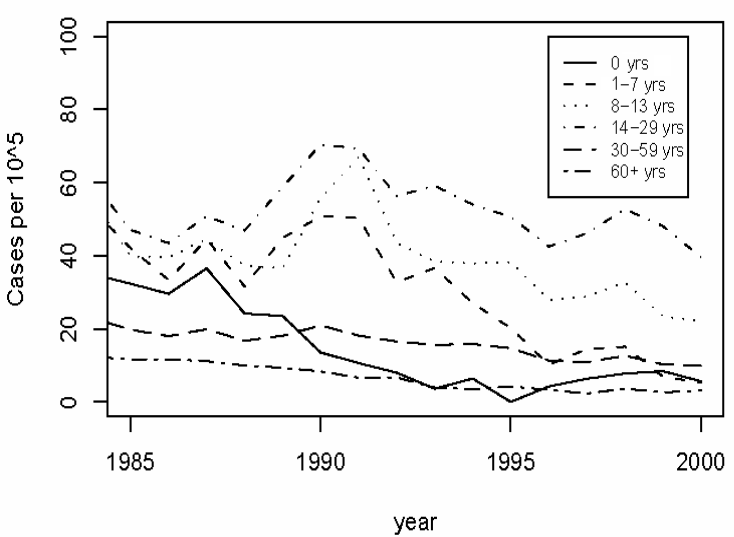

disc model (symptomatic)

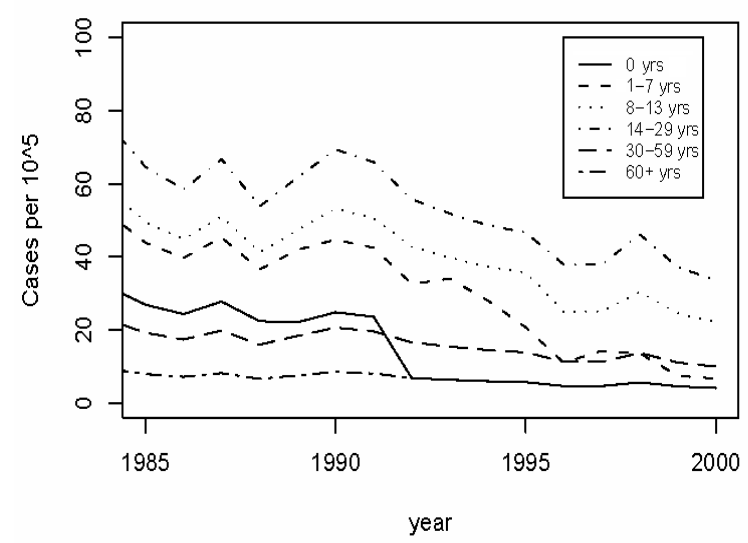

cont model (symptomatic)

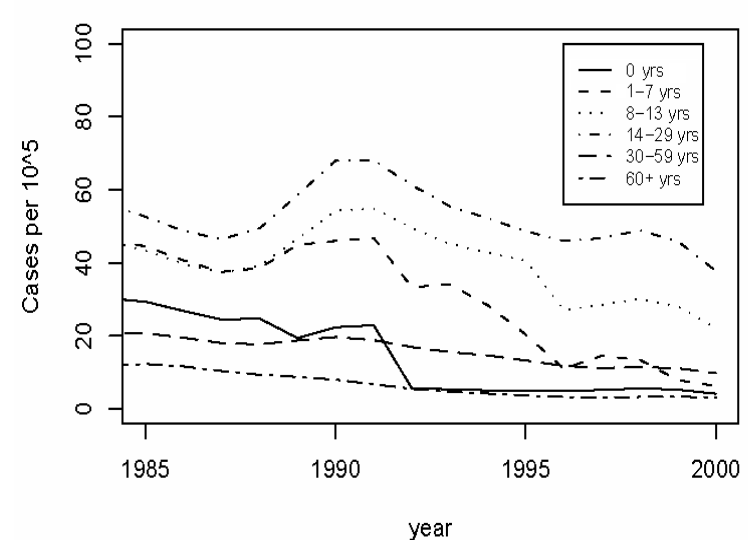

crude (infected)

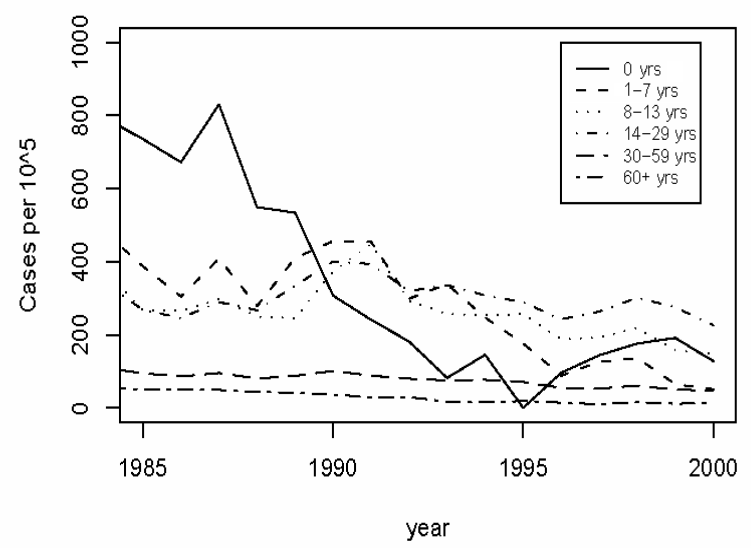

disc model (infected)

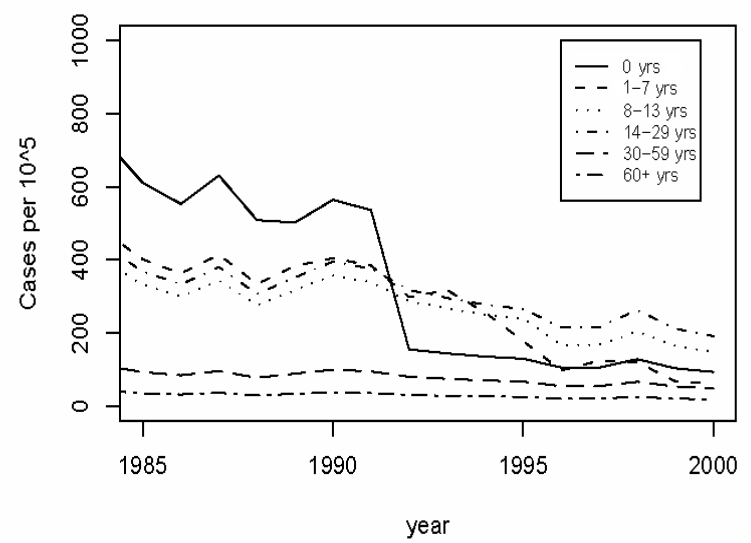

cont model (infected)

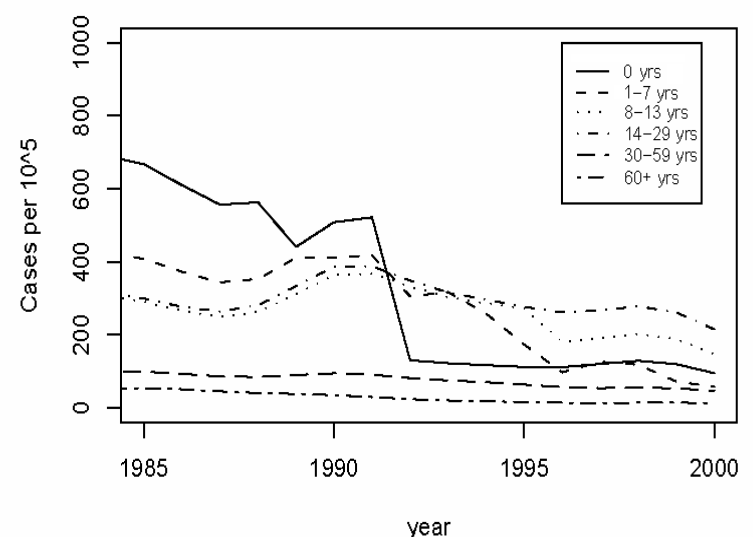

Figure: 2

In parent 3, bin-precise parameter estimates, and 95\%-confidence durations are proven for the commonplace programme for both version (6) (left panel) and model (7) (right panel). Whilst the circle represents the point estimate, the dashed line represents the $95 \%$ confidence c program language period. The usage of the underlying continuous nature of both age and time consequences in an extra dramatic 
immunization impact. The implementation of the mandatory vaccination led to a full-size decrease in prevalence amongst babies of their first year of life with $82 \%$ (based totally on version (7) with $95 \%$ CI: 79\%-84\%), showing the advantages of immunization.

One must take care of the usage of those estimates to make predictions for the situation in which no immunization had occurred. indeed, due to the fact, we do not model the underlying dynamic transmission technique, and our observations are based on acute symptomatic infections most effective, our vaccine-loose estimates do no longer account for all possible components of herd immunity. Herd immunity reduces the force of contamination in non-vaccinated people (i.e. reduces their risk of contamination per unit time), and therefore increases the average age at the contamination of residual infections.[27,28] For hepatitis B, this age shift will cause proportionately more acute infections, however fewer persistent infections.

Our version is based totally on observations related to acute infections most effective, which we inflated to acquire the overall variety of infections, by making use of an impartial age-specific issue to these observations. consequently, our version underestimates the infection-reducing impact of vaccination. but, our version combines age and time precise observations on each incidence and vaccination reputation, we expect this implicit age-associated distortion because of herd immunity to be restricted.
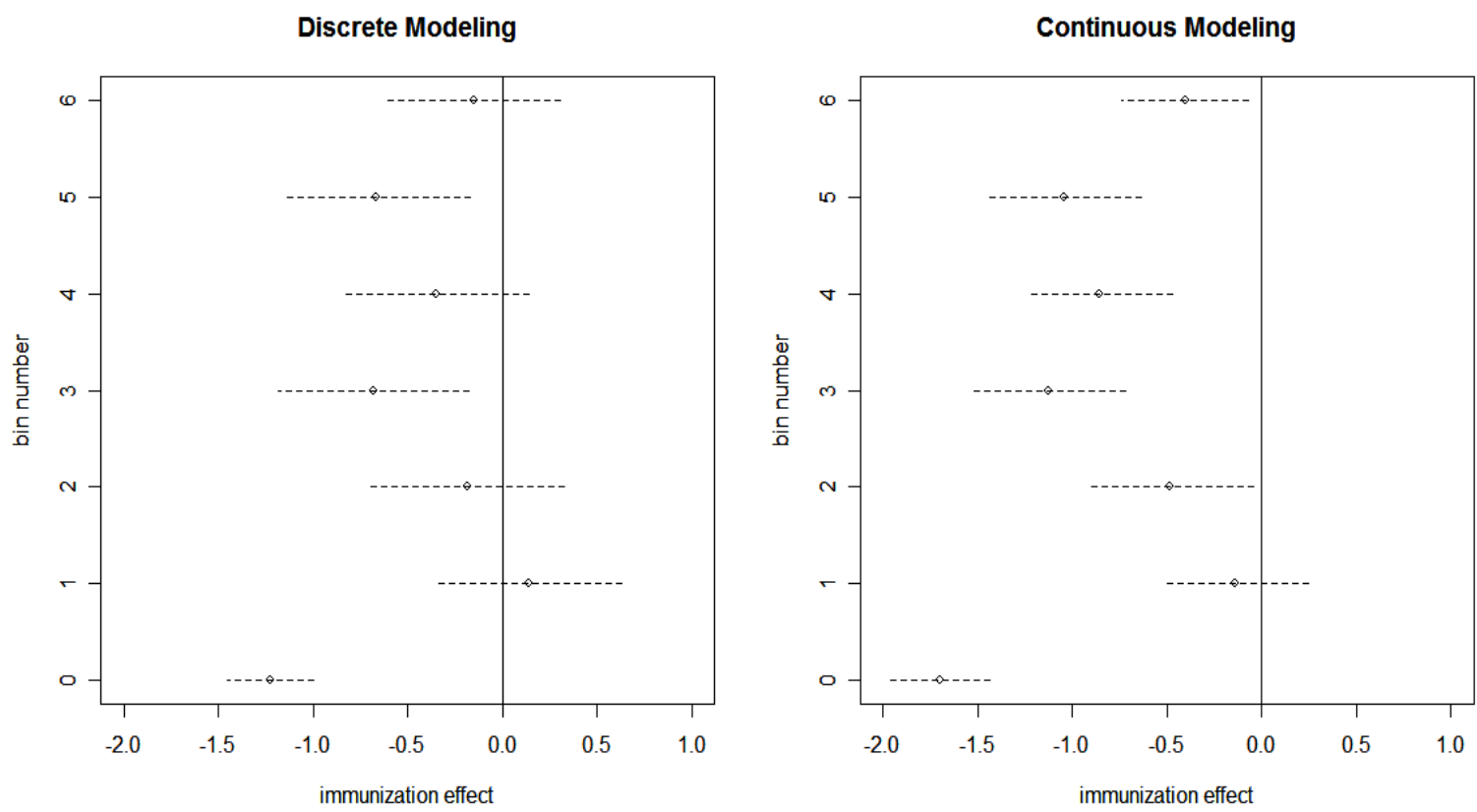

Figure 3

Turning to the infected instances, i.e. correcting for the asymptomatic cases by way of multiplying with age-specific ratios, again model (7) produces smoother curves (figure 2, right panel). The correction for asymptomatic cases clearly shows the advantage of immunization. The range of infected children aged 0 to 112 months decreases highly in 1992, the yr normal immunization programmes commenced. Analogously, decreases over time are observed for the successive age classes showing additionally a herd immunity impact, mainly for the decrease age categories.

\section{VACCINATION FORCE OF INFECTION PROFILE IN BANGLADESH}

Putting immunization effects to zero to mimic the effect of no vaccination, the resulting incidence and susceptibility rates are used to derive the FOI using equations (4) and (5) for different baseline proportions of susceptibility $p_{s}$. In Error! Reference source not found. the estimated year-specific FOI-profiles (conditional on $p_{s}=1$ ) are shown for acute cases (left panel) and infected cases (right panel). Error! Reference source not found. shows aggregated FOI-curves based on different values 
of $p_{s}$, the baseline susceptibility percentage. While $p_{s}$ ranges from 0.2 to 1.0 , the shape of the FOIcurve remains about the same, while the magnitude decreases with increasing percentage susceptibility.

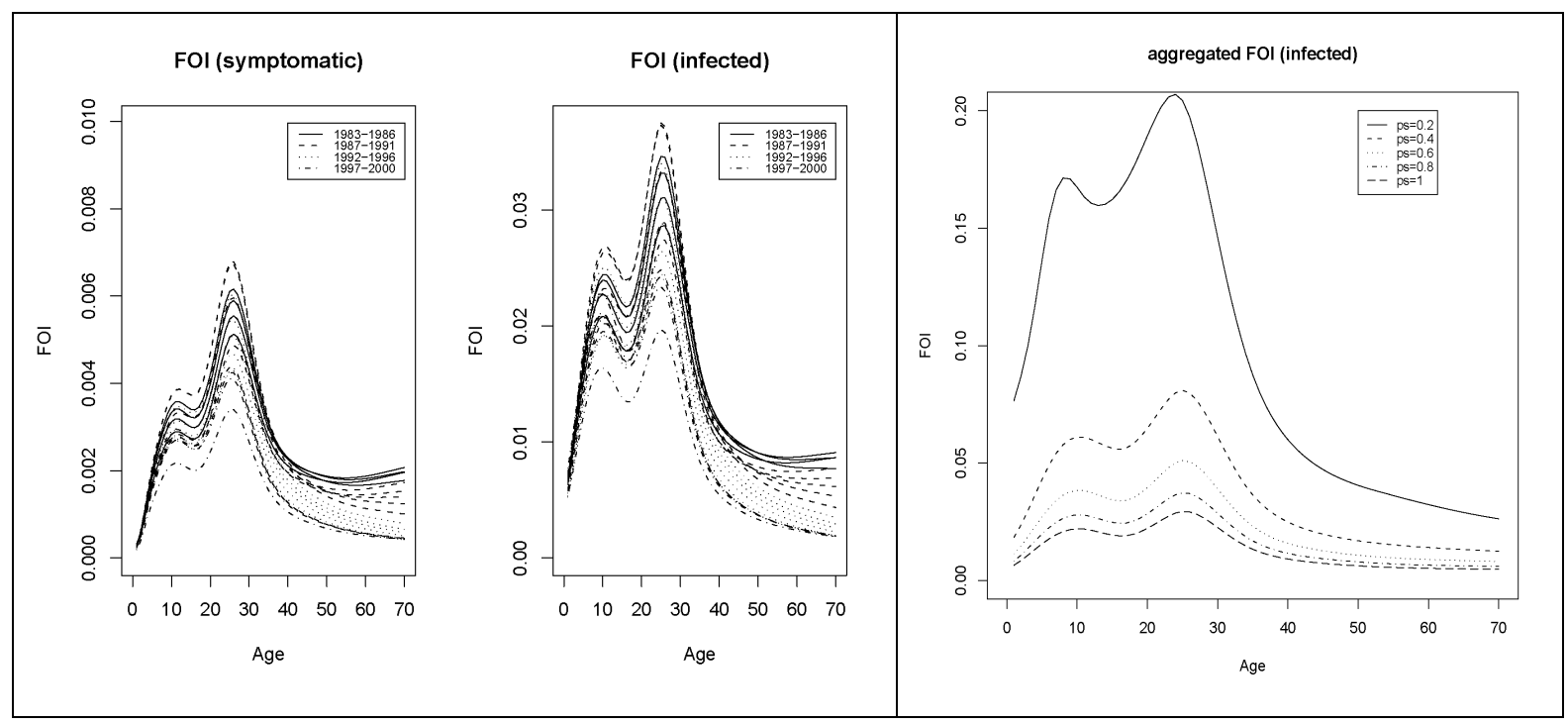

Figure 4

Figure 5

\section{CONCLUSION}

The age-precise FOI-profiles supply two nearby maxima which are placed across the age of nine and 24 , respectively. These findings were also defined in different international locations [29]. The primary nearby most illustrates the significance of horizontal transmission among kids. The second one nearby most shows the importance of sexual transmission and potentially also parenteral transmission by using e.g. needle sharing for injecting drug use among teens.

The significance of horizontal transmission has to not be underestimated and will become specifically apparent whilst staring at the actual FOI (i.e., based totally on the entire wide variety of infections). In this paper, the evolution of Hepatitis B in Bangladesh is defined for the length 2010 till 2019. Bangladesh turned into one of the first nations in Asia that brought mandatory regularly occurring immunization for all new-borns and data recorded protected age-category acute symptomatic instances and population sizes.

This placing is, because correct information of the quantity of acute clinically manifested instances is obtained, making underreporting not an issue. From there, by using the usage of approaches, new to the field of infectious illnesses, now not most effective incidence fees were expected, however additionally qualitative insights inside the FOI had been received. A maximal FOI becomes found around a while nine and 24, illustrating the importance of horizontal transmission among children and of sexual and parenteral (e.g. drug-associated) transmission among young adults. A generalized additive version with penalized splines at the range of acute instances was used to clean over the extraordinary age-classes, exploiting the underlying continuous nature of age and time.

Age-unique ratios had been used to include asymptomatic instances, ensuing in a four to ten-fold boom in occurrence. The proposed approach dietary supplements the present stochastic fashions/time collection which has been proposed to examine case notification information and is, way to present open supply software program, clean to apply. This research illustrates that the hepatitis B vaccination programme in Bangladesh has had a rapid and significant effect on HBV prevalence and therefore may be considered as a success. Moreover, the technique offered on this paper, suggests that case notification records may be used to obtain qualitative insights inside the behaviour of the FOI with age, even inside the presence of a vaccination programme. 


\section{REFERENCES}

Aerts M, Claeskens G, Wand MP. Some theory for penalized spline additive models. Journal of Statistical Planning and Inference 2002; 103: 455-470.

Agresti A. Categorical Data Analysis. Wiley \& Sons, 2002.

Akaike H. Information theory and an extension of the maximum likelihood principle. In: Petrov B, Csaki F. (eds.) Budapest: Akademia Kiado 1973: 267-281.

Anderson RM, May RM. Age-related changes in the rate of disease transmission: implications for the design of vaccination programmes. J Hyg (Lond) 1985; 94: 365-435.

Beutels P et al. Hepatitis B in St Petersburg, Russia (1994-1999): incidence, prevalence and force of infection. Journal of Viral Hepatitis 2003; 10: 141-149.

Craven P, Wahba G. Smoothing noisy data with spline functions. Numerische Mathematik 1979; 31: 377-403.

Currie I, Durban M, Eilers P. Smoothing and forecasting mortality rates.Statistical Modelling 2004; 4: 279-298.

Edmunds WJ, Medley GF, Nokes DJ. The influence of age on the development of the hepatitis B carrier state. In: Proceedings of the Royal Society of London. Series B, Biological Sciences 1993; 253: 197-201

Fine PEM. Herd immunity: history, theory, practice. Epidemiol Rev 1993; 15: 265-302.

FizSimons D et al. Strengthening immunization systems and introduction of hepatitis B vaccine in central and Eastern Europe and the Newly Independent States. Viral Hepatitis Prevention Board, University of Antwerp 2005.

Frome EL, Checkoway H. Epidemiologic programs for computers and calculators. Use of Poisson regression models in estimating incidence rates and ratios. American Journal of Epidemiology 1985; 121: 309-323.

Hastie T Tibshirani R. Generalized additive models: some applications. Journal of the American Statistical Association 1987; 82: 371-386.

Hastie T, Tibshirani R. Generalized Additive Models. Chapman and Hall 1990.

Held L, Hofmann M, Höhle M, Schmid V. A two component model for counts of infectious diseases. Biostatistics 2006; 7: 422-437.

Held L, Höhle M, Hofmann M. A statistical framework for the analysis of multivariate infectious disease surveillance data. Statistical Modelling 2005; 5: 187-199.

Huang C, Wang Y, Li X, Ren L, Zhao J, Hu Y, et al. Clinical features of patients infected with 2019 HBV, China. Lancet. 2020.

Li Q, Guan X, Wu P, Wang X, Zhou L, Tong Y, et al. Early transmission dynamics in Wuhan, China, of HBV infected pneumonia. N Engl J Med. 2020.

Marx BD, Eilers PHC. Direct generalized additive modelling with penalized likelihood. Computational Statistics and Data Analysis 1998; 28: 193-209.

McCullagh P, Nelder J. Generalized Linear Models. Chapman \& Hall 1989.

McMahon BJ, Alward WLM, Hall DB. Acute hepatitis B virus infection: relation of age to the clinical expression of disease and subsequent development of the carrier state. Journal of Infectious Diseases 1985; 15: 604-609.

RDC Team R: A language and environment for statistical computing 2004.

Shapiro CN. Epidemiology of hepatitis B. Pediatric Infectious Disease Journal 1993; 12: 443-447.

Smith L, Hyndman RJ, Wood SN. Spline interpolation for demographic variables: the monotonicity problem. Journal of population research 2004; 21 : 95-98.

Thurston S, Wand M, Wiencke J. Negative binomial additive models.Biometrics 2000; 56: 139-144.

Wahba G. Spline bases, regularization, and generalized cross validation for solving approximation problems with large quantities of noisy data. In: Cheney, W. (ed.) Approximation Theory III, Academic Press 1980: 905-912.

Wahba G. Spline Models for Observational Data CBMS-NSF series. In: SIAM, Philadelphia, 1990.

Wallinga $\mathbf{J}$ et al. Estimation of measles reproduction ratios and prospects for elimination of measles by vaccination in some Western European countries. Epidemiology and Infection 2001; 127: 281-295.

Wood SN. mgcv: GAMs and Generalized Ridge Regression for R. R News 2001; 1: 20-25.

Wood SN. Modelling and Smoothing Parameter Estimation with Multiple Quadratic Penalties. Journal of the Royal Statistical Society, Series B 2000; 62: 413-428.

Wood SN. Stable and efficient multiple smoothing parameter estimation for generalized additive models. Journal of the American Statistical Association, 2005.

Wood SN. Thin Plate Regression Splines. Journal of the Royal Statistical Society 2003; 65: 95-114.

World Health Organization. World Health Organization cited January 19, 2020. Zhou P, Yang XL, Wang XG, Hu B, Zhang L, Zhang W, et al. A pneumonia outbreak associated with a new HBV of probable bat origin. Nature. 2020 . 